\title{
Genetic testing and diagnosis of inherited retinal diseases
}

\author{
Byron L. Lam ${ }^{1 *}$ (D) Bart P. Leroy ${ }^{2,3}$, Graeme Black ${ }^{4,5,6}$, Tuyen Ong ${ }^{7}$, Dan Yoon ${ }^{8}$ and Karmen Trzupek ${ }^{9}$
}

\begin{abstract}
Inherited retinal diseases (IRDs) are a diverse group of degenerative diseases of the retina that can lead to significant reduction in vision and blindness. Because of the considerable phenotypic overlap among IRDs, genetic testing is a critical step in obtaining a definitive diagnosis for affected individuals and enabling access to emerging gene therapy-based treatments and ongoing clinical studies. While advances in molecular diagnostic technologies have significantly improved the understanding of IRDs and identification of disease-causing variants, training in genetic diagnostics among ophthalmologists is limited. In this review, we will provide ophthalmologists with an overview of genetic testing for IRDs, including the types of available testing, variant interpretation, and genetic counseling. Additionally, we will discuss the clinical applications of genetic testing in the molecular diagnosis of IRDs through case studies.
\end{abstract}

Keywords: Inherited retinal disease, Ophthalmology, Molecular diagnosis, Genetic testing, Genetic counseling, Case studies, Next-generation sequencing

\section{Introduction}

Inherited retinal diseases (IRDs) are a heterogenous group of visually debilitating diseases caused by pathogenic variation in proteins critical to retinal function. The majority of IRDs are characterized by retinal degeneration, which can lead to significant vision impairment and blindness [1-4]. Collectively, IRDs are estimated to affect more than 2 million people worldwide [5, 6]. Because of the phenotypic overlap of several IRDs, establishing a definitive clinical diagnosis may be difficult [7]. Therefore, molecular genetic testing has become an important strategy to complement clinical findings and confirm or clarify a diagnosis.

With an increased understanding of the human genome and the wide scope of genetic variants identified to be associated with IRDs ( $>250$ causative genes) [8], emerging genetic testing technologies such as nextgeneration sequencing are allowing clinicians to better

\footnotetext{
*Correspondence: blam@med.miami.edu

${ }^{1}$ Bascom Palmer Eye Institute, University of Miami Miller School of Medicine, 900 NW 17th Street, Miami, FL 33156, USA

Full list of author information is available at the end of the article
}

diagnose IRDs [9-11]. The advent of these sophisticated testing technologies for genetic disorders has highlighted the need for broader awareness of human genetics and its relevance to personalized medicine in IRDs.

The American Academy of Ophthalmology Task Force on Genetic Testing and the European Reference Network for Rare Eye Diseases recommend genetic testing for all individuals with presumed or suspected IRDs for which a causative gene or genes have been identified [12, 13]. However, most clinical ophthalmologists are unfamiliar with genomic diagnostics. In a retrospective analysis of genetic testing utilization for individuals with IRDs within a large, university-based health system conducted from 2008 to 2018, providers felt that genetic testing was useful in IRD management [14]. However, the analysis found that genetic testing was infrequently utilized, with only $1.5 \%$ of 207 individuals with IRDs undergoing genetic testing ordered from an ophthalmologist's office [14].

Historically, genetic testing has been ordered and interpreted by IRD specialists and ocular genetic counselors at large academic research centers [12]. However, this structure does not adequately meet patient demand for

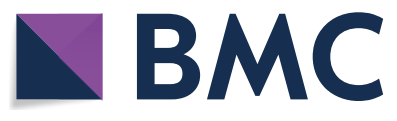

(c) The Author(s) 2021. Open Access This article is licensed under a Creative Commons Attribution 4.0 International License, which permits use, sharing, adaptation, distribution and reproduction in any medium or format, as long as you give appropriate credit to the original author(s) and the source, provide a link to the Creative Commons licence, and indicate if changes were made. The images or other third party material in this article are included in the article's Creative Commons licence, unless indicated otherwise in a credit line to the material. If material is not included in the article's Creative Commons licence and your intended use is not permitted by statutory regulation or exceeds the permitted use, you will need to obtain permission directly from the copyright holder. To view a copy of this licence, visit http://creativecommons.org/licenses/by/4.0/. The Creative Commons Public Domain Dedication waiver (http://creativeco mmons.org/publicdomain/zero/1.0/) applies to the data made available in this article, unless otherwise stated in a credit line to the data. 
3 major reasons. First, there are not enough IRD specialists and ocular genetic counselors at academic medical centers to meet patient demand for genetic testing. While there are currently close to 5000 certified genetic counselors in the United States, $<1 \%$ of them have expertise in ophthalmology [15]. Second, many individuals with IRDs are geographically isolated from those centers and unable or unwilling to travel. It is important for affected individuals to be evaluated by a retina specialist who is familiar with IRDs and has the expertise to make a provisional clinical diagnosis. Community-based retina specialists have successfully incorporated genetics into their practice by ordering testing themselves; however, the importance of both pre- and post-test genetic counseling should be recognized. Particularly in the United States, telemedicine-based genetic counseling services are becoming more widely available to support geographically or economically disadvantaged individuals in accessing specialists in ocular genetics [16]. This allows community-based retina specialists to partner with telephone-based genetic counselors to help disclose results to individuals and manage conversations regarding complex results and risks to family members. Lastly, the global use of genetic testing is limited because of the challenge for the budgets and structure of regional healthcare systems to cover the cost of genetic testing, particularly in countries with low resources [13].

Early and accurate diagnosis is necessary for individuals with IRDs to enable patient decision-making, to identify suitable clinical studies or treatment opportunities, and to improve patient outcomes. The objective of this review is to provide a general understanding of genetic testing and its clinical applications for IRDs through case studies.

\section{Genetic testing}

Genetic testing is the analysis of an individual's DNA to detect genetic changes or variants that could lead to disease [17]. With the application of improved molecular testing technology, the likelihood of identifying a causative variant in individuals with IRDs has increased [10, $11,18-20]$. Identifying the disease-causing variant can not only illuminate or confirm a diagnosis but can also improve medical management by informing prognosis, reducing the need for additional electrophysiologic testing, clarifying guidance in ocular surveillance, and advising appropriate changes in therapies and/or supplementation [19]. Genetic testing also allows for accurate identification of inheritance pattern, thereby improving genetic counseling for affected individuals and their families [19]. Additionally, with an approved retinal gene therapy for biallelic RPE65 mutation-associated retinal dystrophy, Luxturna ${ }^{\circledR}$ (voretigene neparvovec-rzyl)
[21, 22], and several ocular gene therapy clinical studies in progress [23-25], confirming a molecular diagnosis through genetic testing may help individuals access the latest treatment options or qualify for study participation $[2,7]$.

When a person has been clinically diagnosed with a presumed IRD, diagnostic genetic testing is indicated. At this time, genetic testing should not be used to rule out an IRD, since a negative result from large panel genetic tests could reflect the limits of the panel's design and not the comprehensive spectrum of potential genetic candidates [12]. In many cases, diagnostic testing is recommended on the basis of physical symptoms or findings from clinical examination. If an individual is asymptomatic but a disease-causing molecular variant has previously been confirmed in a family member, genetic testing can be used for predictive testing or carrier testing [26]. Predictive testing is used to detect genetic mutations in individuals before they present with symptoms to assess future risk of disease $[12,26]$. When no therapeutic intervention exists, predictive testing should be approached with caution, particularly in children. For many IRDs, reduced penetrance or a wide degree of variability in clinical symptoms is known to occur and can complicate the interpretation of results for asymptomatic individuals. The American College of Medical Genetics and Genomics and the American Academy of Pediatrics explicitly discourage the routine testing of at-risk minors for adultonset conditions with no available therapy or change in management [27]. Carrier testing is used to identify individuals who carry a single pathogenic variant in a recessive or X-linked disease gene that, when present with another pathogenic variant, can cause genetic disease. Carrier testing may be performed on individuals who do not present with symptoms but may be at risk for passing a genetic disease on to their children [26].

Genetic tests can assess single genes or, more commonly, panels of genes associated with a group of genetic diseases [2]. Previously, genetic testing was primarily performed on a single-gene basis, where the small number of genes tested were those determined to be most likely associated with disease on the basis of clinical assessment $[7,19,28]$. This often produced a low diagnostic yield [7]. While traditional single-gene sequencing may be sufficient for diagnosing IRDs with mostly only one diseaseassociated gene identified, such as congenital anirdia (PAX6 gene), systematic testing of single genes may be inefficient for diagnosing more complex IRDs that have a high degree of genetic heterogeneity, such as retinitis pigmentosa (> 100 identified causative genes) $[2,7,8,12,19$, $29]$. With the introduction of next-generation sequencing, testing multiple genes in a single assay has become possible [9]. Next-generation sequencing sequences 
millions of DNA fragments in parallel and matches them to a reference genome using bioinformatics to detect variants [30, 31]. Next-generation sequencing can be used to completely sequence an individual's DNA (whole genome sequencing) or to sequence just the protein-coding regions (whole exome sequencing) [30-32]. In the clinical assessment of IRDs, next-generation sequencing can also be used to constrain sequencing to just the coding regions of genes known to cause retinal diseases using a targeted multigene panel [30, 31].

In the processing of genetic test results, identified variants are analyzed to determine their potential association with an IRD phenotype. Not all identified variants cause disease [33]. Genetic variant interpretation, as defined by the American College of Medical Genetics, is classified using a 5-class system (Table 1) [33, 34]. Variants may be classified as benign on the basis of several criteria, including well-established functional data showing no damaging effect of the variant on protein function or splicing, lack of segregation in affected members of a

Table 1 Summary of reported variant classification and interpretation [34]

\begin{tabular}{ll}
\hline Result & Variant interpretation \\
\hline Benign & Clearly not disease-causing \\
Likely benign & Unlikely to be disease-causing \\
Uncertain significance & $\begin{array}{l}\text { Evidence is insufficient to support or reject } \\
\text { pathogenicity, and additional data are } \\
\text { needed }\end{array}$ \\
Likely pathogenic & Likely to be disease-causing \\
Pathogenic & Clearly disease-causing \\
\hline
\end{tabular}

family, and variant frequency in the general population [34]. Variants classified as "likely benign" are those with an estimated $>90 \%$ certainty of being benign [34]. Pathogenic variants are disease-causing mutations [34]. Variants classified as "likely pathogenic" are those with an estimated $>90 \%$ certainty of causing disease [34]. If a variant does not fulfill benign or pathogenic criteria, or if the evidence for benign and pathogenic are contradictory, the variant defaults to being classified as uncertain significance [34]. Variants of uncertain significance should be reported with caution, and additional testing or iterative clinical investigations may be required to assess the effects of these variants [34]. Processing of genetic findings should always be done in the context of the clinical phenotype [35].

\section{Case study 1}

A 60-year-old healthy male with progressive night visual impairment and peripheral visual loss for many years and a diagnosis of choroideremia was referred for evaluation as a participant for a choroideremia natural history study. Family history was negative. Best-corrected visual acuity was 20/50 in each eye. Funduscopic findings showed areas of well-demarcated chorioretinal atrophy, with small islands of retained functional area, in the macula of each eye (Fig. 1). The working diagnosis was choroideremia; the genetic testing with next-generation sequencing panel showed the individual was negative for pathogenic mutations of the CHM gene and positive for 2 novel heterozygous mutations of the C2orf71 gene, namely, p.Arg1202Ter (nonsense mutation) and p.Ser134ArgfsTer47 (frameshift mutation). Mutations of
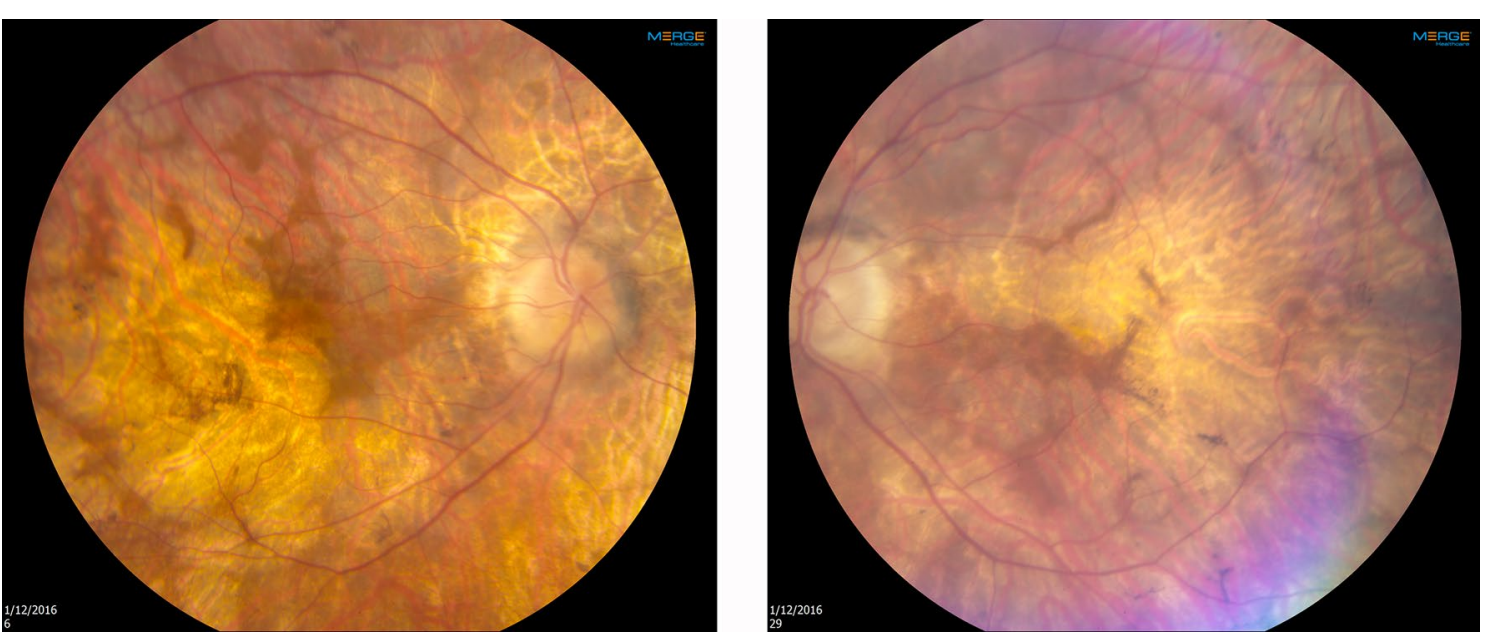

Fig. 1 Fundoscopic images from a 60-year-old healthy male with a clinical diagnosis of choroideremia. The genetic testing with next-generation sequencing panel showed the individual was negative for pathogenic mutations of the CHM gene and positive for 2 novel heterozygous mutations of the C2orf71 gene 
the $C 2$ orf71 gene cause autosomal recessive retinitis pigmentosa; the 2 novel $C 2 o r f 71$ genetic variants found were considered likely positive. The individual was counseled regarding the hereditary pattern and the prognosis of his C2orf71-associated IRD and will be considered a candidate for future C2orf71 clinical trials.

Pedigree analyses are an important complement to molecular assessment of IRDs [36, 37]. These analyses can support the results of clinical phenotyping, inform reproductive risks, and help guide genetic testing [38]. However, it is important to consider that some IRDs can be difficult to predict on the basis of family history alone. For example, approximately 50\% of individuals with retinitis pigmentosa have no known family history of the disease [31, 39], and although most will have autosomal recessive inheritance, up to $10 \%$ may have $\mathrm{X}$-linked inheritance and approximately $24 \%$ will have dominant inheritance with no known affected family members as the result of reduced penetrance or a de novo genetic variant [39]. Therefore, different patterns of inheritance and associated causative genes should not be ruled out without molecular confirmation.

\section{Case study 2}

A 7-year-old male suspected of difficulty with night vision was found to have $20 / 20$ vision bilaterally with normal appearing maculas (Fig. 2). He reported no vision problems and has 2 brothers who were asymptomatic. The individual's next-generation sequencing panel genetic testing showed a hemizygous pathogenic mutation in the ORF15 region of the RPGR gene on the $\mathrm{X}$ chromosome (c.2270_2271delAG; p.Glu757GlyfsTer12; this means a deletion of nucleotides $\mathrm{A}$ and $\mathrm{G}$ at positions 2270 and 2271 in the exon leading to a complete change

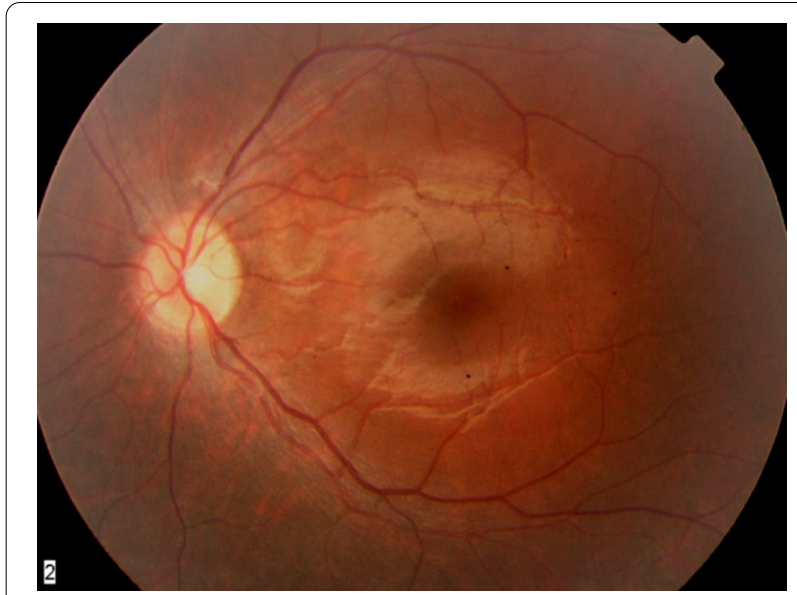

Fig. 2 Funduscopic images from 7-year-old boy with a clinical diagnosis of RPGR-mutated X-linked retinitis pigmentosa in amino acid sequence starting at the change of position 757 from glutamic acid to glycine until an early termination after 12 changed amino acids; see https://varno men.hgvs.org/recommendations/protein/variant/frame shift/ for description of mutation nomenclature). Subsequent testing of his siblings showed a similar genotype. The genetic testing revealed that all 3 siblings actually had X-linked retinitis pigmentosa due to homozygous mutations in the RPGR gene. After 16 years, at the age of 23 years, the individual had a vision of $20 / 40$ bilaterally with significant constricted visual fields and was enrolled into a clinical trial for $R P G R$ gene therapy.

\section{Genetic counseling}

Prior to coordination of genetic testing, a provider familiar with the genetics of retinal diseases (often an IRD specialist or an ocular genetic counselor) should outline the benefits, limitations, and potential implications of genetic testing with affected individuals and their caregivers $[37,40]$. For example, individuals should be made aware that targeted genetic testing may miss some differential diagnoses because of phenotypic overlap among various IRDs [7, 41]. Conversely, it should also be considered that broader testing strategies may increase the likelihood of unexpected or unclear results and that conditions that seem isolated may actually be syndromic [7]. It is important for individuals to understand that receiving genetic testing does not guarantee that they will receive a molecular diagnosis for their IRD, and a positive genetic test result will not necessarily qualify them for a clinical trial or therapy [37]. As not all of the genes and variants associated with IRDs have been identified, testing may not detect the disease-causing variant for all individuals [37]. After the pretest consultation with the counselor, individuals must indicate informed consent to proceed with genetic testing [36].

\section{Case study 3}

A 59-year-old female was referred for genetic counseling of her IRD. She noticed significant night blindness at age 17 and was diagnosed with retinitis pigmentosa at age 28 . Further, at the age of 28 , she was diagnosed with polycystic kidney disease and underwent a unilateral kidney transplant 2 days later. Genetic testing with a limited panel of 31 genes implicated in early onset retinal disease was ordered before referral for genetic counseling and indicated that she was positive for a heterozygous pathogenic variant in the RPE65 gene $(\mathrm{c} .11+5 \mathrm{G}>\mathrm{A})$. The ordering provider incorrectly interpreted the positive test result as diagnostic of RPE65-related IRD, changed the diagnosis to Leber congenital amaurosis, and advised her that she would likely qualify for treatment with Luxturna. 
During the genetic counseling appointment, the individual disclosed that she had 2 brothers who died of kidney disease in late childhood. Subsequent additional genetic testing revealed a complete deletion of the NPHP1 gene (interpreted as pathogenic) in the homozygous state. The individual's retinal disease, kidney disease, and family history are consistent with a syndromic form of retinal dystrophy due to the NPHP1 gene. The presence of a pathogenic variant in the RPE65 gene indicates that she is additionally a carrier of recessively inherited RPE65-related retinal dystrophy, a state that does not qualify her for Luxturna gene therapy.

Post-test genetic counseling is also important for interpreting and discussing genetic test results in light of patient history $[19,37]$. Accurate interpretation of genetic test results is very important and helps health care providers advise individuals about their prognosis and provide more clarity about how an eye disease is inherited [12]. As the number of genes commercially available for sequencing increases and the use of genetic sequencing processes becomes more widespread, accurate interpretation of the results from these tests is critical [34]. On the basis of findings from the genetic test, counselors will also advise on the implications of results for at-risk family members, offer appropriate screening or preventative strategies, and educate on other potential health or lifestyle effects $[37,40]$. As previously discussed, results from genetic testing can help providers direct individuals to available targeted therapies and/or establish eligibility for participation in applicable ongoing clinical studies [19].

\section{Case study 4}

A female with progressive impairment of night vision from late childhood, legal blindness by age 16, and a diagnosis of severe nonsyndromic retinitis pigmentosa was referred for evaluation. Genetic testing showed that she was positive for 2 heterozygous mutations of the $B B S 1$ gene, p.Met390Arg (pathogenic) and p.Glu224Lys (likely pathogenic), resulting in a diagnosis of Bardet-Biedl syndrome. This condition is associated with multiple risks of other health conditions, requiring significant medical follow-up. The genetic counselor provided medical management guidelines established for Bardet-Biedl syndrome to the patient, the referring retina specialist, and the primary care physician. These include regular ophthalmic evaluations, body mass index calculations, diabetes testing, and lipid profiling.

Barriers to genetic testing remain a challenge for individuals with IRDs and health care professionals. One significant challenge for the widespread implementation of genetic testing is the varied payment/ reimbursement mechanisms across countries [42, 43]. In some countries, there is limited coverage of genetic testing costs $[1,42]$. Insurance companies and other funding agencies may be reluctant to pay for genetic testing unless there is clear evidence that the results will affect medical management [31]. Another significant barrier is limited patient access to expert providers and genetic testing services, especially in underserved and remote areas [44]. Without guidance from an IRD or genetics expert, ordering and interpretation of genetic tests can be complex. Because of the functional limitations associated with having an IRD, affected individuals may need a caregiver to facilitate access to genetic testing services and appointments with genetic counselors or IRD specialists. This could result in loss of workdays or working time for both the affected individual and caregiver [45]. Another challenge is ophthalmologists' generally limited knowledge of genetic services, genetic conditions, and patient risk factors [44]. This lack of knowledge can lead to insufficient referrals from ophthalmologists to IRD experts and appropriate genetic services. Inadequate completion of family history analysis can also impede genetic testing efforts [44]. While a thorough family history can aid decision-making and inform genetic testing approaches, incomplete assessment may delay testing or lessen the likelihood of identifying a causative mutation. Finally, difficulties consolidating genetic data into public databases can also be a hurdle for genetic testing $[1,46,47]$. While large databases are commonly used by diagnostic laboratories, entries may not be complete or up to date $[46,47]$. Variant data may be stored in local or specialized databases and not collated in a multicenter database that can be easily accessed or shared $[46,47]$.

Despite the complexity of genetic tests and their practical limitations, it is necessary to overcome these barriers to ensure that the best medical care is provided for individuals affected by IRDs. Some potential approaches to overcome these barriers and improve the patient's experience include expanding education of health care professionals about IRDs and genetic testing options, creating tools to connect affected individuals with experts in IRDs [48], and improving data sharing via strategies to support streamlined and secure distribution of genomic variant data from clinical laboratories [1]. A valuable service that has transformed health care in recent years is telemedicine [16]. Telemedicine can facilitate medical education, e-health patient monitoring, and patient consultation through which ocular genetic counselors can provide remote counseling to overcome geographical constraints and expand access to patient care $[16,49]$. 


\section{Genetic testing recommendations for individuals with IRDS}

To enhance genetic disease management, a decision tree algorithm may be useful for easy reference in clinical practice to help determine the indications for molecular diagnosis in individuals with IRDs (Fig. 3). The American Academy of Ophthalmology recommendations for the genetic testing of IRDs state that whenever clinical findings suggest the possibility for an IRD for which a causative gene or genes have been identified, the treating ophthalmologist should either discuss the value of genetic testing and order an appropriate test (if available) or refer the individual to an IRD specialist or ocular genetic counselor with expertise in the selection and interpretation of molecular tests [12].

Retina specialists or ocular genetic counselors will review the individual's clinical, ocular, and family history [37]. In some cases, additional clinical assessments may be advised. If evaluation supports the diagnosis of an IRD and genetic testing is considered appropriate after pretest counseling, a genetic test will be ordered. If a pathogenic variant is identified, the specialist or counselor will assess the result in the context of the individual's family and clinical history to confirm diagnosis and will provide actionable recommendations including medical management strategies. Ideally, individuals with IRDs should be

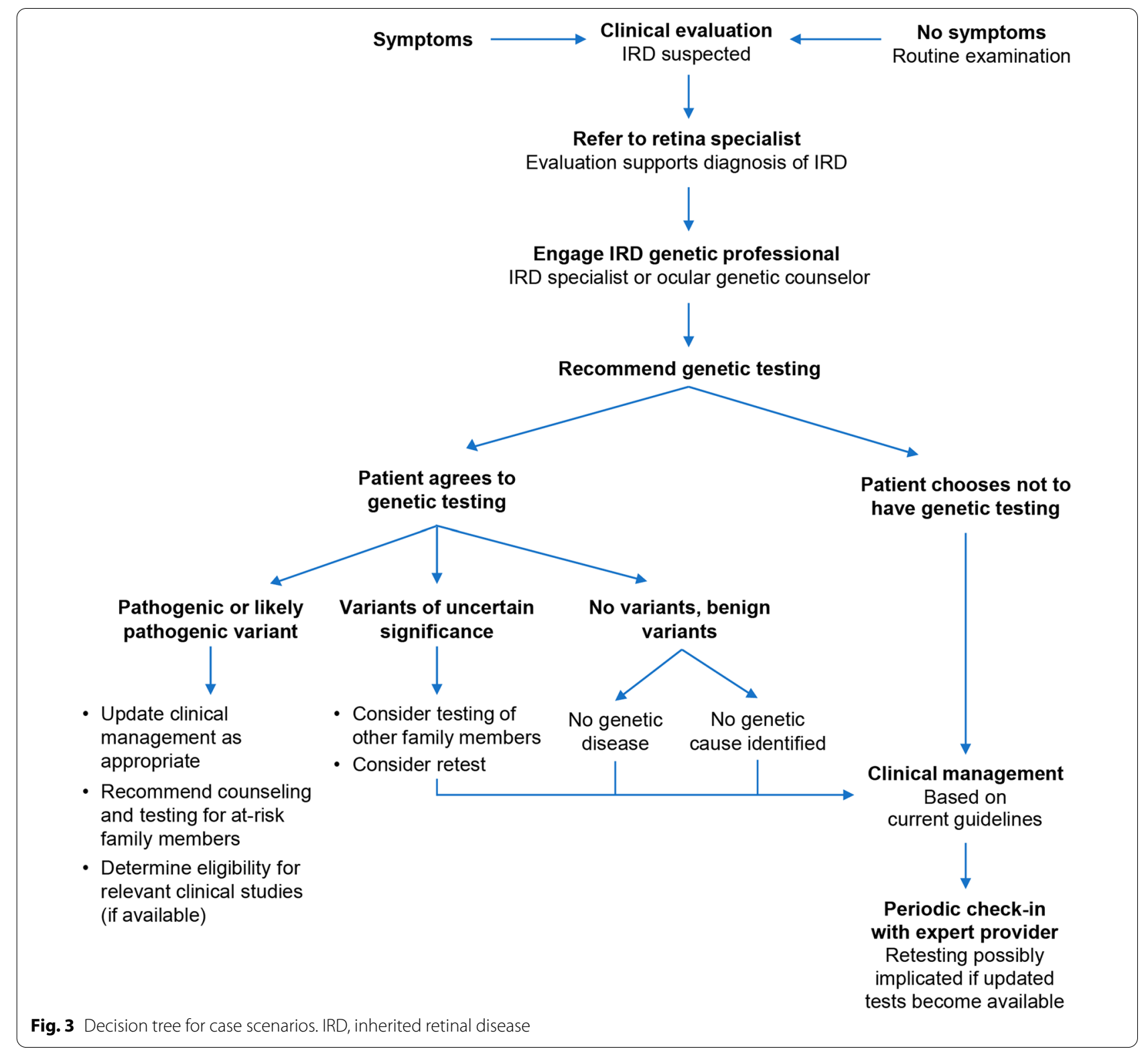


Table 2 Useful websites

\begin{tabular}{ll}
\hline Website & Description \\
\hline $\begin{array}{l}\text { GeneReviews } \\
\text { https://www.ncbi.nlm.nih.gov/books/NBK1116/ }\end{array}$ & $\begin{array}{l}\text { Provides detailed information on clinical scenarios and specific single-gene disorders, } \\
\text { authored by experts. These articles also contain recommendations on basic manage- } \\
\text { ment, genetic counseling, and genetic testing }\end{array}$ \\
$\begin{array}{l}\text { VisionServe Alliance } \\
\text { https://visionservealliance.org/vision-loss-resources/lost-your- } \\
\text { vision/ }\end{array}$ & \begin{tabular}{l} 
Provides a listing of local support services for those with vision impairment or blindness \\
\hline
\end{tabular}
\end{tabular}

assessed annually for associated, treatable findings such as cataracts and cystoid macular edema. Individuals with IRDs are also advised to return for follow-up expeditiously if there is an acute change in vision.

If testing reveals a variant of uncertain significance, additional clinical data may be collected or family members may be tested; however, this does not always help to elucidate the effect of the variant [34]. Variants of uncertain significance are almost always found with next-generation sequencing panel testing or whole exome sequencing, with or without applied filters. Family member testing can be pursued for parental or segregation analysis if additional family members are available to test. The absence of a genetic variant in both parents of a child can prove de novo inheritance, which provides strong evidence of pathogenicity. In dominant and $\mathrm{X}$-linked conditions, if unequivocal co-segregation of a clinical phenotype with a variant is established in at least 3 generations, then a variant of uncertain significance is highly likely to be pathogenic and reclassification of a Class 3 (variant of uncertain significance) to Class 4 or 5 (pathogenic or likely pathogenic) is warranted. Further, identification of gene-specific phenotype features (e.g., flat electro-oculography in a patient with vitelliform dystrophy and a BEST1 variant) can also support variant interpretation. If phenotypic clinical findings suggest more than one specific genotype, the variant of unknown significance can sometimes be reassessed on the basis of the effect on gene and protein structure and function to determine potential pathogenicity. Direct assays may be useful in cases in which variants are thought to alter RNA splicing; however, functional assessments may be timeconsuming and expensive. Structural assessments such as in silico analysis may be valuable; however, they should generally only be used to support other lines of evidence.

If test results are negative for pathogenic variants, this could either indicate that the individual does not have a genetic cause of their disease [50] or, more often the case, that the molecular cause of the disease has not been identified. It is possible that individuals receiving a negative genetic test result may harbor variants in currently unknown IRD genes or in regions of known genes not currently identified using current testing methodologies.
Genetic reevaluation may be valuable and, because new pathogenic genetic variants are continually being identified, may be considered every $2-5$ years. Retesting may be dependent on the mode of genetic analysis initially utilized. Reanalysis of existing data (e.g., in cases in which patients have had whole exome or whole genome analysis) may be simpler and cost less than a complete reevaluation. In cases in which a new genetic test is conducted, it is important to assess whether it will be substantially different than previous analyses, as diagnostic tests may not be updated regularly. In all cases, coordination between the primary provider and retina specialist, and/or genetic counselor, is critical to facilitate genetic testing and guide appropriate care for individuals with IRDs. Table 2 provides some useful websites regarding basic management of genetic disorders, as well as genetic counseling, genetic testing, and available supportive services.

\section{Conclusions}

Inherited retinal diseases present challenges in molecular diagnostics because of their high genetic heterogeneity, overlapping clinical presentations, and variability in inheritance patterns. Currently, ophthalmologists who come across individuals experiencing symptoms consistent with an IRD may benefit from the expertise of an ocular genetic counselor or IRD specialist. With the complexity of IRDs, collaboration with specialists may allow for improved decision-making regarding the most appropriate genetic test panel, pre- and post-test counseling, and accurate interpretation of the test findings in the context of the clinical diagnosis. While adequate genetic testing resources and specialty providers may be unavailable in many regions, improved understanding of the genetics within ophthalmic subspecialties and increased use of telemedicine-based options may help meet the needs of individuals to fully understand their diagnosis.

\section{Acknowledgements}

Medical writing and editorial assistance were provided by Bethany Reinecke, PhD, MedThink SciCom, Cary, NC, and Gina Uhlenbrauck, ELS, under the direction of the authors with funding support from Biogen.

\section{Authors' contributions}

TO was responsible for the conceptualization of the review and literature search. BLL and KT provided the included case studies. BLL, BPL, DY, and KT 
were major contributors to the drafting of the manuscript. All authors read and approved the final manuscript.

\section{Funding}

Biogen provided funding for medical writing and editorial assistance pertaining to the drafting of the manuscript under the direction of the authors.

\section{Availability of data and materials}

Data sharing is not applicable to this article as no data sets were generated or analyzed during the current study.

\section{Declarations}

\section{Ethics approval and consent to participate}

Not applicable.

\section{Consent for publication}

Consent for publication of deidentified patient clinical and genetic information has been obtained where applicable.

\section{Competing interests}

$B L L$ has received research and clinical trial funding from the National Eye Institute, AGTC, Biogen, Editas Medicine, Pixium Vision, and ProQR and consultancy fees from Biogen and ProQR. BPL has received consultancy fees from Bayer, GenSight Therapeutics, IVERIC Bio, Novartis Pharma International \& Belgium, Spark Therapeutics, ProQR Therapeutics, REGENXBIO, and Vedere Bio; has received travel support from GenSight Therapeutics, IVERIC Bio, Novartis Pharma International \& Belgium, Spark Therapeutics, and ProQR Therapeutics; and reports an unpaid consultancy with LookoutGTx. GB has received consultancy fees from GenSight Therapeutics and Novartis Pharma International. TO has nothing to disclose. DY is an employee and stockholder of Biogen. KT has received an honorarium from Biogen for speaking at an internal training event.

\section{Author details}

1 Bascom Palmer Eye Institute, University of Miami Miller School of Medicine, 900 NW 17th Street, Miami, FL 33156, USA. ²Department of Ophthalmology, Ghent University and Ghent University Hospital, Ghent, Belgium. ${ }^{3}$ Ophthalmic Genetics and Visual Electrophysiology, Division of Ophthalmology, Children's Hospital of Philadelphia, Philadelphia, PA, USA. ${ }^{4}$ UK Inherited Retinal Disease Consortium, Manchester, UK. ${ }^{5}$ Genomics England Clinical Interpretation Partnership, Manchester, UK. ${ }^{6}$ Manchester Centre for Genomic Medicine, St. Mary's Hospital, Manchester, UK. ${ }^{7}$ Ring Therapeutics, Cambridge, MA, USA. ${ }^{8}$ Biogen, Cambridge, MA, USA. ${ }^{9}$ Ocular and Rare Disease Genetics Services, InformedDNA, St Petersburg, FL, USA.

\section{Received: 12 July 2021 Accepted: 28 November 2021}

Published online: 14 December 2021

\section{References}

1. Duncan JL, Pierce EA, Laster AM, Daiger SP, Birch DG, Ash JD, et al. Inherited retinal degenerations: current landscape and knowledge gaps. TransI Vis Sci Technol. 2018;7:6.

2. American Academy of Ophthalmology. Recommendations on clinical assessment of patients with inherited retinal degenerations. 2016. https://www.aao.org/clinical-statement/recommendations-on-clinicalassessment-of-patients. Accessed November 20, 2020.

3. Patricio MI, Barnard AR, Xue K, MacLaren RE. Choroideremia: molecular mechanisms and development of AAV gene therapy. Expert Opin Biol Ther. 2018;18:807-20

4. Lipinski DM, Thake M, MacLaren RE. Clinical applications of retinal gene therapy. Prog Retin Eye Res. 2013;32:22-47.

5. Hanany M, Rivolta C, Sharon D. Worldwide carrier frequency and genetic prevalence of autosomal recessive inherited retinal diseases. Proc Natl Acad Sci U S A. 2020;117:2710-6.

6. Berger W, Kloeckener-Gruissem B, Neidhardt J. The molecular basis of human retinal and vitreoretinal diseases. Prog Retin Eye Res. 2010;29:335-75.
7. Lee K, Garg S. Navigating the current landscape of clinical genetic testing for inherited retinal dystrophies. Genet Med. 2015;17:245-52.

8. RetNet: Summaries of genes and loci causing retinal disease. https://sph. uth.edu/RETNET/sum-dis.htm\#A-genes. Accessed 20 Nov 2020.

9. Chiang JP, Trzupek K. The current status of molecular diagnosis of inherited retinal dystrophies. Curr Opin Ophthalmol. 2015;26:346-51.

10. Wang $F$, Wang $H$, Tuan $H F$, Nguyen $D H$, Sun $V$, Keser $V$, et al. Next generation sequencing-based molecular diagnosis of retinitis pigmentosa: identification of a novel genotype-phenotype correlation and clinical refinements. Hum Genet. 2014;133:331-45.

11. O'Sullivan J, Mullaney BG, Bhaskar SS, Dickerson JE, Hall G, O'Grady A, et al. A paradigm shift in the delivery of services for diagnosis of inherited retinal disease. J Med Genet. 2012;49:322-6.

12. Stone EM, Aldave AJ, Drack AV, Maccumber MW, Sheffield VC, Traboulsi E, et al. Recommendations for genetic testing of inherited eye diseases: report of the American Academy of Ophthalmology task force on genetic testing. Ophthalmology. 2014;119:2408-10.

13. Black GC, Sergouniotis P, Sodi A, Leroy BP, Van Cauwenbergh C, Liskova P, et al. The need for widely available genomic testing in rare eye diseases: an ERN-EYE position statement. Orphanet J Rare Dis. 2021;16:142.

14. Neiweem AE, Hariprasad SM, Ciulla TA. Genetic testing prevalence, guidelines, and pitfalls in large, university-based medical systems. Ophthalmic Surg Lasers Imaging Retina. 2021;52:6-10.

15. National Society for Genetic Counselors. https://www.nsgc.org/page/ about-nsgc. Accessed 20 Nov 2020

16. Rathi S, Tsui E, Mehta N, Zahid S, Schuman JS. The current state of teleophthalmology in the United States. Ophthalmology. 2017;124:1729-34.

17. McPherson E. Genetic diagnosis and testing in clinical practice. Clin Med Res. 2006;4:123-9.

18. Eisenberger T, Neuhaus C, Khan AO, Decker C, Preising MN, Friedburg $C$, et al. Increasing the yield in targeted next-generation sequencing by implicating CNV analysis, non-coding exons and the overall variant load: the example of retinal dystrophies. PLoS ONE. 2013;8:e78496.

19. Trzupek KM. Where genetic testing fits in the retina practice. https:// www.retina-specialist.com/article/where-genetic-testing-fits-in-theretina-practice. Accessed 20 Nov 2020.

20. Consugar MB, Navarro-Gomez D, Place EM, Bujakowska KM, Sousa ME, Fonseca-Kelly ZD, et al. Panel-based genetic diagnostic testing for inherited eye diseases is highly accurate and reproducible, and more sensitive for variant detection, than exome sequencing. Genet Med. 2015;17:253-61.

21. Luxturna [package insert]. Spark Therapeutics Inc; 2017.

22. FDA approves hereditary blindness gene therapy. Nat Biotechnol. 2018:36:6. https://doi.org/10.1038/nbt0118-6a.

23. Trapani I, Auricchio A. Seeing the light after 25 years of retinal gene therapy. Trends Mol Med. 2018:24:669-81.

24. Hulliger EC, Hostettler SM, Kleinlogel S. Empowering retinal gene therapy with a specific promoter for human rod and cone ON-bipolar cells. Mol Ther Methods Clin Dev. 2020;17:505-19.

25. Garafalo AV, Cideciyan AV, Heon E, Sheplock R, Pearson A, Yu CW, et al. Progress in treating inherited retinal diseases: early subretinal gene therapy clinical trials and candidates for future initiatives. Prog Retin Eye Res. 2020;77:100827.

26. U.S. National Library of Medicine. National Institutes of Health. Department of Health \& Human Services. Help me understand genetics. https:// medlineplus.gov/download/genetics/understanding/testing.pdf. Accessed 20 Nov 2020

27. Committee on Bioethics, Committee on Genetics, American College of Medical Genetics and Genomics Social, Ethical, Legal Issues Committee. Ethical and policy issues in genetic testing and screening of children. Pediatrics. 2013;131:620-2.

28. Newman WG, Black GC. Delivery of a clinical genomics service. Genes (Basel). 2014;5:1001-17.

29. Landsend ECS, Lagali N, Utheim TP. Congenital aniridia-a comprehensive review of clinical features and therapeutic approaches. Surv Ophthalmol. 2021;66:1031-50.

30. Behjati S, Tarpey PS. What is next generation sequencing? Arch Dis Child Educ Pract Ed. 2013;98:236-8.

31. Moore AT. Genetic testing for inherited retinal disease. Ophthalmology. 2017:124:1254-5 
32. Tatour Y, Ben-Yosef T. Syndromic inherited retinal diseases: genetic, clinical and diagnostic aspects. Diagnostics (Basel). 2020;10:779.

33. Tsang SH, Sharma T. Genetic testing for inherited retinal dystrophy: basic understanding. Adv Exp Med Biol. 2018;1085:261-8.

34. Richards S, Aziz N, Bale S, Bick D, Das S, Gastier-Foster J, et al. Standards and guidelines for the interpretation of sequence variants: a joint consensus recommendation of the American College of Medical Genetics and Genomics and the Association for Molecular Pathology. Genet Med. 2015;17:405-24

35. Stone EM, Andorf JL, Whitmore SS, DeLuca AP, Giacalone JC, Streb LM, et al. Clinically focused molecular investigation of 1000 consecutive families with inherited retinal disease. Ophthalmology. 2017;124:1314-31.

36. Mejecase C, Malka S, Guan Z, Slater A, Arno G, Moosajee M. Practical guide to genetic screening for inherited eye diseases. Ther Adv Ophthalmol. 2020;12:2515841420954592.

37. Foundation for Fighting Blindness. Genetic testing for retinal degenerative diseases. https://www.fightingblindness.org/genetic-testing-for-retin al-degenerative-diseases-information-and-resources-for-affected-indiv iduals-families-and-health-care-providers. Accessed 21 Nov 2020.

38. Stroh E. Taking the family history in genetic disease: a guide for ophthalmologists. Curr Opin Ophthalmol. 2011;22:340-6.

39. Bravo-Gil N, Gonzalez-Del Pozo M, Martin-Sanchez M, Mendez-Vidal C, Rodriquez-de la Rua E, Borrego S, et al. Unravelling the genetic basis of simplex retinitis pigmentosa cases. Sci Rep. 2017;7:41937.

40. Blain D, Brooks BP. Molecular diagnosis and genetic counseling in ophthalmology. Arch Ophthalmol. 2007;125:196-203.

41. Lionel AC, Costain G, Monfared N, Walker S, Reuter MS, Hosseini SM, et al. Improved diagnostic yield compared with targeted gene sequencing panels suggests a role for whole-genome sequencing as a first-tier genetic test. Genet Med. 2018;20:435-43.

42. Vozikis A, Cooper DN, Mitropoulou C, Kambouris ME, Brand A, Dolzan V, et al. Test pricing and reimbursement in genomic medicine: towards a general strategy. Public Health Genomics. 2016;19:352-63.

43. Mansfield BC, Yerxa BR, Branham KH. Implementation of a registry and open access genetic testing program for inherited retinal diseases within a non-profit foundation. Am J Med Genet C Semin Med Genet. 2020;184:838-45.

44. Delikurt T, Williamson GR, Anastasiadou V, Skirton H. A systematic review of factors that act as barriers to patient referral to genetic services. Eur J Hum Genet. 2015;23:739-45.

45. National Academies of Sciences, Engineering, and Medicine; Health and Medicine Division; Board on Health Sciences Policy; Roundtable on Genomics and Precision Health. Exploring the barriers to accessing genomic and genetic services. In: Understanding disparities in access to genomic medicine: proceedings of a workshop. Washington, DC: National Academies Press; 2018. https://www.ncbi.nlm.nih.gov/books/ NBK538442/. Accessed 21 Nov 2020.

46. van Huet RA, Oomen CJ, Plomp AS, van Genderen MM, Klevering BJ, Schlingemann RO, et al. The RD5000 database: facilitating clinical, genetic, and therapeutic studies on inherited retinal diseases. Invest Ophthalmol Vis Sci. 2014;55:7355-60.

47. Chiang J, Gorin MB. Challenges confronting precision medicine in the context of inherited retinal disorders. Exp Rev Precis Med Drug Dev. 2016;1:195-205.

48. Leroy B. Symposium conducted at: European Society of Retina Specialists Meeting 2020; October 2-4, 2020; Virtual.

49. Kay C. Logistics of genetic testing: an overview for retina specialists. Retin Physician. 2017;14:55-8.

50. Centers for Disease Control and Prevention. Genetic testing. https://www. cdc.gov/genomics/gtesting/genetic_testing.htm. Accessed 1 Dec 2020.

\section{Publisher's Note}

Springer Nature remains neutral with regard to jurisdictional claims in published maps and institutional affiliations.

Ready to submit your research? Choose BMC and benefit from:

- fast, convenient online submission

- thorough peer review by experienced researchers in your field

- rapid publication on acceptance

- support for research data, including large and complex data types

- gold Open Access which fosters wider collaboration and increased citations

- maximum visibility for your research: over 100M website views per year

At BMC, research is always in progress.

Learn more biomedcentral.com/submissions 